\title{
Efecto de la temperatura y la precipitación durante el llenado de grano sobre la dormición y sensibilidad al agua en granos de cebada (Hordeum vulgare $\mathrm{L}_{\text {.) }}$ )
}

\author{
González, S. N., Viega, L. M., Beretta, A., Córdoba, M. A. y Rossi, C. A.
}

\begin{abstract}
RESUMEN
Los granos de cebada (Hordeum vulgare L.) pueden presentar dormición y sensibilidad al agua poscosecha, que retrasan su utilización industrial. Ambas, dependen del genotipo y del ambiente durante la maduración del grano. Con el fin de analizar el efecto de la temperatura media y las precipitaciones acumuladas durante y en el tercio final del llenado de grano, sobre la dormición y la sensibilidad al agua y la relación entre ellas, fueron sembrados tres cultivares de cebada en cuatro fechas durante el 2009 y dos durante el 2011. Se registraron temperaturas y precipitaciones diarias desde antesis a madurez fisiológica. La dormición se evaluó como la germinación a los 12 días posmadurez fisiológica y la sensibilidad al agua como la germinación en exceso de agua, a los 100 días pos-madurez fisiológica. Las variables que presentaron mejor correlación con germinación y sensibilidad al agua fueron la temperatura media y las precipitaciones acumuladas durante el llenado de grano. Ambientes con mayores temperaturas medias durante el llenado de grano disminuyeron la dormición, pero la disminución de la sensibilidad al agua dependió del cultivar. El incremento de las precipitaciones durante el llenado de grano disminuyó la dormición, pero aumentó la sensibilidad al agua en los tres cultivares.
\end{abstract}

Palabras clave: análisis; efectos ambientales; germinación; Hordeum vulgare; malta.

González, S. N., Viega, L. M., Beretta, A., Córdoba, M. A. and Rossi, C. A. 2019. Effect of temperature and precipitation during grain filling on dormancy and water sensitivity in barley grains (Hordeum vulgare L.). Agriscientia 36 (2): 19-27

\section{SUMMARY}

After harvesting, barley grains (Hordeum vulgare L.) can show dormancy and water sensitivity, which delays industrial use. Both effects depend on the barley 
genotype and environmental conditions during grain maturation. In order to analyze the effect of the average temperature and the total rainfall accumulated during and in the final third of the grain filling, on the dormancy and water sensitivity and the relationship between them, three cultivars of barley were sownin four dates in 2009 and two in 2011 . Temperature and daily rainfall were recorded from anthesis to physiological maturity. The dormancy was evaluated as germination at 12 days post physiological maturity and the water sensitivity as germination in water excess conditions at 100 days post physiological maturity. The variables that presented a higher correlation with germination and water sensitivity were average temperature and accumulated rainfall during grain maturity. Environments with average high temperature during grain filling, reduced dormancy, but on water sensitivity decrease was cultivar dependent. The increase of rainfall during grain filling, decreased dormancy, but increased water sensitivity in all three cultivars.

Keywords: analysis; environmental effects; germination; Hordeum vulgare,malt.

González, S. N. y Rossi, C. A.: Instituto Nacional de Investigación Agropecuaria, Ruta $50 \mathrm{~km} \mathrm{11,} \mathrm{CP} 70$ 000. Colonia, Uruguay. Viega, L. M.: Universidad de la República, Facultad de Agronomía, Garzón 780, CP 12 900. Montevideo, Uruguay. Beretta, A.: Instituto Nacional de Investigación Agropecuaria, Laboratorio de Suelos, Plantas y aguas. Córdoba, M. A.: Universidad Nacional de Córdoba, Facultad de Ciencias Agropecuarias. Félix Marrone 746, Ciudad Universitaria, CP 5000, Córdoba, Argentina. Correspondencia a: sngonzalez@inia.org.uy

\section{INTRODUCCION}

Los granos de cebada cervecera (Hordeum vulgare L.) pueden presentar dormición y sensibilidad al agua al momento de la cosecha. Estas características retrasan su utilización industrial para la producción de malta, debido a que es necesario un período de almacenamiento prolongado para que el grano alcance su máxima germinación y vigor (Arias, 1991). A los efectos de disminuir el período de almacenamiento sería deseable que los cultivares en uso no presenten dormición. Sin embargo, un cierto grado de dormición es necesario para prevenir el pregerminado en espiga, en años con exceso de lluvia durante el período de cosecha (Reuss, Cassells y Green, 2003).

La dormición consiste en un bloqueo de la germinación en condiciones adecuadas de temperatura, agua y oxígeno; la sensibilidad al agua es la disminución de la germinación en condiciones de exceso de agua (Benech-Arnold, Giallorenzi, Frank y Rodríguez, 1999). Cuando se maltean granos con dormición y sensibilidad al agua, la germinación es lenta y despareja (BenechArnold, Gualano, Leymarie, Côme y Corbineau, 2006), lo cual reduce el rendimiento y la calidad de la malta.

La dormición en granos de cebada está condicionada por las glumelas (el lema y la pálea), que tienen gran cantidad de componentes fenólicos, cuya oxidación por enzimas polifenoloxidasas reduce el suministro de oxígeno al embrión (Côme, 1982). Este mecanismo incide en el catabolismo del ácido abscísico (ABA), hormona inhibidora de la germinación y la biosíntesis de giberelinas (Gas), promotoras de la germinación (Millar et al., 2006; Liu, Ye, Liu, Chen y Zhang, 2010). La capacidad de consumo de oxígeno de las glumelas decrece durante el almacenamiento y consecuentemente también la dormición (Bewley y Black, 1994; Gong, Li, Zhou, Bonnardeaux y Yang, 2014). Este proceso puede tardar meses, con lo cual se incrementan los costos de producción de malta.

La dormición en cebada es controlada por muchos genes y fuertemente influenciada por el ambiente (Castro, Benítez, Hayes, Viega y Wright, 2010; Gong et al., 2014). En Uruguay, Benítez (1999) reportó que incrementos de la temperatura media del aire desde la antesis a la madurez fisiológica redujeron de diferente forma la dormición en cinco genotipos de cebada cervecera. Sin embargo, otros estudios limitaron el efecto de la temperatura en la dormición de los granos de cebada en la última etapa del llenado de grano. En este sentido, Rodríguez, González, Insausti, Margineda y Benech-Arnold (2001) reportaron que aumentos de la temperatura media del aire de 300 a 350 grados 
días (GDD) en el tercio final del llenado de grano incrementaron el índice de germinación a los 12 días posteriores a madurez fisiológica $\left(r^{2}=0,95\right)$ en el cultivar Quilmes Palomar. Similarmente, Gualano (2004) detectó que la sensibilidad a la temperatura se localizó en la última etapa del llenado (275-325 GDD) para tres cultivares de cebada. Estudios más recientes (Castro et al., 2010) reportaron una estrecha relación $\left(r^{2}=0,52\right)$ entre la temperatura media del tercio final del Ilenado de grano y el nivel de dormición de los granos de 100 líneas dobles haploides derivados del cruzamiento BCD47 $x$ Baronesse.

Las temperaturas durante el llenado de grano modulan la dormición a cosecha debido a que regulan el contenido y/o la sensibilidad del embrión al ABA (Benech-Arnold, 2004). Por otro lado, en los cultivares de cebada B1215 y Quilmes Palomar, la tasa en que los granos perdieron su dormición se correlacionó con diferencias en el contenido y sensibilidad del embrión al ABA (Benech-Arnold et al., 1999). Según estos autores, después de la madurez fisiológica, los embriones del cv. B1215 comenzaron a reducir su contenido de ABA y a perder la sensibilidad a éste mucho antes que los del cv. Quilmes Palomar.

Existen escasos estudios del efecto de las precipitaciones durante el llenado de grano en la dormición. En años con lluvias por debajo del promedio durante esta etapa, un cultivar de cebada con dormición moderada puede presentar una reducción de la dormición e incrementar los riesgos de daño por pregerminado en el campo (Benech-Arnold, 2002). En cambio, se reportó que la sequía impuesta durante la misma etapa incrementó la dormición en cebada (Aspinall, 1965) y en trigo (Biddulph, Plummer, Setter y Mares, 2007). Por otra parte, la sensibilidad a la disponibilidad hídrica podría limitarse, como en el caso de la temperatura, a la última etapa del llenado de grano y no a todo el período del mismo (Gualano, 2004).

Los mecanismos responsables de la sensibilidad al agua permanecen desconocidos, pero guardan estrecha relación con la dormición. En este sentido, Castro et al. (2010) estudiaron las bases genéticas de la dormición y de la sensibilidad al agua en una población de mapeo de 100 líneas doble haploides de BCD47/Baronesse y comprobaron que ambas características tienen una misma base genética pero no detectaron correlación entre ellas. Al igual que la dormición, la sensibilidad al agua está fuertemente influenciada por el ambiente durante la maduración del grano, y su importancia es mayor cuando se presentan condiciones climáticas frías y húmedas durante el llenado de grano (Jansson, 1962). En condiciones de exceso de agua los microorganismos incrementan su actividad (Briggs y McGuinness, 1993; Thomas y Usher, 2001), forman un biofilm que restringe la difusión de oxígeno al interior del grano, y en consecuencia inducen sensibilidad al agua (Gaber y Roberts, 1969; Noots, Delcour y Michiels, 1999). Es así que Woonton, Jacobsen, Sherkat y Stuart (2005) observaron que en granos con presencia de microorganismos la dormición durante el almacenamiento disminuyó, pero la sensibilidad al agua permaneció elevada.

Los objetivos de este trabajo fueron, analizar el efecto de la temperatura media y las precipitaciones acumuladas durante y en el tercio final del llenado de grano, sobre la dormición y la sensibilidad al agua y la relación entre ellas, en tres cultivares de cebada.

\section{MATERIALES Y MÉTODOS}

Se seleccionaron tres cultivares de cebada de dos hileras con diferentes ciclos de maduración y sensibilidad al agua: INIA Ceibo de ciclo largo (IC) cultivar caracterizado por su mayor sensibilidad al agua, INIA Guaviyú de ciclo corto (IG) y Maltería Pampa de ciclo largo (MP), ambos de sensibilidad al agua menores con relación a IC (Suburú et al., 2007; 2008). Los tres cultivares fueron sembrados en el campo experimental de INIA La Estanzuela, Colonia, Uruguay $\left(57^{\circ} 41^{\prime} \mathrm{O}, 34^{\circ} 20^{\prime} \mathrm{S}\right)$, en cuatro fechas de siembra durante el año 2009 (26 de mayo, 25 de junio, 14 de julio y 8 de agosto) y dos fechas de siembra durante el año 2011 (31 de mayo y 15 de agosto). Los experimentos se instalaron con un diseño de parcelas en bloques completos al azar con tres repeticiones en 2009 y cuatro repeticiones en 2011; la siembra se realizó con sembradora autopropulsada de siembra directa de 6 surcos a $15 \mathrm{~cm}$ entre surcos y con una población de 250 semillas viables por $\mathrm{m}^{2}$. Se registraron datos de la temperatura media diaria del aire $\left({ }^{\circ} \mathrm{C}\right)$ y la precipitación acumulada $(\mathrm{mm})$ desde antesis hasta madurez fisiológica para cada tratamiento.

El tiempo térmico (TT) acumulado durante el período de llenado de grano, fue calculado como la suma de los valores de temperatura media diaria del aire $\left(T_{m d}\right)$ por encima de una temperatura base $\left(T_{b}\right)$ de $5,5^{\circ} \mathrm{C}$ (Pararajasingham y Hunt, 1991):

$$
\mathrm{TT}_{\mathrm{A}-\mathrm{MF}}=\sum_{\mathrm{d}=\mathrm{A}}^{\mathrm{MF}}\left(\mathrm{T}_{\mathrm{md}}-\mathrm{T}_{\mathrm{b}}\right)
$$

donde $d$ es un día en el intervalo de tiempo 
comprendido entre antesis (A) y madurez fisiológica (MF)

El tiempo térmico acumulado se evaluó entre la antesis y la madurez fisiológica. Para determinar la temperatura y la precipitación acumulada en el último tercio (UT), el TT se dividió en tres intervalos desde la antesis hasta la madurez fisiológica, con iguales acumulación de tiempo térmico. Los valores de Tmd fueron obtenidos de la estación meteorológica de INIA La Estanzuela. Al momento de antesis (aristas visibles por encima de la hoja bandera) y cuando el $50 \%$ de las plantas de cada parcela se encontraban en dicho estado, se identificaron con cintas de color 350 espigas provenientes de tallos principales (Rodríguez et al., 2001). Cuando las espigas llegaron a madurez fisiológica (pérdida del $100 \%$ del color verde de las glumas y el pedúnculo) fueron cosechadas y trilladas manualmente conformándose una muestra compuesta para cada tratamiento.

\section{Evaluación de dormición}

A los 12 días posteriores a madurez fisiológica se estudió la dormición de los granos mediante el comportamiento germinativo (Rodríguez et al., 2001). Para cada parcela, dos submuestras de 100 granos fueron colocados a germinar en cajas de Petri de $9 \mathrm{~cm}$ de diámetro, sobre dos hojas de papel de filtro Whatman $\mathrm{N}^{\circ} 1$ y $4 \mathrm{ml}$ de agua destilada a $20 \pm 2{ }^{\circ} \mathrm{C}$ y $90 \%$ de humedad relativa, en oscuridad durante 7 días (Castro et al., 2010). La germinación fue evaluada en función de los granos que presentaron protrusión de radícula aproximadamente de 2 a $3 \mathrm{~mm}$ (Benech-Arnold, Gualano, Leymarie, Côme y Corbineau 2006).

\section{Evaluación de sensibilidad al agua}

Las muestras fueron almacenadas en sacos de lienzo a temperatura ambiente $\left(20-22{ }^{\circ} \mathrm{C}\right)$ en las instalaciones del Laboratorio de Análisis de semillas de INIA La Estanzuela por un período de 100 días, momento en el cual se determinó la sensibilidad al agua a través del test de Pollock (1962). Para cada repetición se tomó una muestra de 500 semillas, divididas en 5 repeticiones de 100 granos cada una. Al igual que en el ensayo de dormición, se colocaron en cajas de Petri con 2 hojas de papel filtro $\mathrm{N}^{\circ} 1$ de $90 \mathrm{~mm}$ de diámetro. A dos de las submuestras del ensayo anterior se les agregaron $4 \mathrm{ml}$ de agua, y a los tres restantes, $8 \mathrm{ml}$ de agua. Posteriormente se colocaron las placas en gabinete de germinación con temperatura de $20 \pm 2{ }^{\circ} \mathrm{C}$ y $90 \%$ de humedad relativa en oscuridad.
Se realizaron recuentos de semillas con protrusión de radícula al segundo, tercer y cuarto día. A partir de ello se estimó el valor de sensibilidad al agua (\%) como la diferencia entre el promedio de las semillas germinadas en el test de $4 \mathrm{ml}$ y el obtenido en el de $8 \mathrm{ml}$, (ambos a las 72 horas).

Se realizó un análisis de la varianza para evaluar el efecto del cultivar y ambiente (definido como la combinación de mes y año de siembra), en la germinación y la sensibilidad al agua; se consideró heterogeneidad de varianza en el efecto del cultivar. Se verificó la existencia de efecto de cultivar y se realizó el ANAVA para cada cultivar para evaluar el efecto ambiente. Se correlacionaron (coeficiente de Pearson) las variables climáticas: temperatura media desde antesis y madurez fisiológica $\left(\mathrm{T}_{\mathrm{A}-\mathrm{MF}}\right)$; precipitación acumulada desde antesis y madurez fisiológica $\left(\mathrm{P}_{\mathrm{A}-\mathrm{MF}}\right)$; temperatura media en el último tercio del llenado de grano( $\left.\mathrm{T}_{\mathrm{UT}}\right)$; precipitación acumulada en el último tercio del llenado de grano $\left(P_{U T}\right)$, entre sí y con las variables germinación y sensibilidad al agua. Se eligieron las variables ambientales que presentaron mejor correlación con germinación y sensibilidad al agua, para ajustar un modelo lineal por cultivar. Se ajustó un modelo lineal para estimar sensibilidad al agua en función de la germinación para cada cultivar. La información fue analizada con el software InfoStat/P (Di Rienzo et al., 2016).

\section{RESULTADOSY DISCUSIÓN}

En los dos años de experimentos las $T_{\text {A-MF }} y$ $T_{U}$, fueron mayores con el atraso en la fecha de siembra. La diferencia de estos valores entre cultivares se debió a diferencias entre ellos en el momento de antesis. En el año 2009, para el cv. IC y el cv. MP, las fechas de siembra de agosto registraron un incremento de la $T_{A-M F}$ de 3 a $4{ }^{\circ} \mathrm{C}$ con respecto a las de mayo, mientras que para el cv. IG este incremento fue de $6{ }^{\circ} \mathrm{C}$ (Tabla 1).

En el año 2011 la $T_{\text {A-MF }}$ se incrementó en $4{ }^{\circ} \mathrm{C}$ para el cv. IC y el cv. MP y $3{ }^{\circ} \mathrm{C}$ para el cv. IG. Con respecto a las condiciones en el último tercio, en el año 2009, para los cv. IC y MP las fechas de siembra de mayo registraron similar $T_{U T}$ que las de agosto, mientras que para el cv. IG este incremento fue de $4^{\circ} \mathrm{C}$. En el año 2011 la $T_{\text {UT }}$ se incrementó 4 a $5{ }^{\circ} \mathrm{C}$ para los $\mathrm{Cv}$. IC e IG y $3^{\circ} \mathrm{C}$ para el cv. MP por atrasos en la fecha de siembra.

Las $\mathrm{P}_{\text {A-MF }}$ promedio en 2009 fueron mayores (230 $\mathrm{mm}$ ) a las de 2011 (62 mm) (Tabla 1). En 2009, para los tres cultivares, las fechas de siembra de mayo registraron en promedio mayores $P_{\text {A-MF }}(316 \mathrm{~mm})$ con respecto a las de agosto (103 $\mathrm{mm})$. En el año 
Tabla 1. Fecha de siembra, temperatura media y precipitación acumulada, desde antesis a madurez fisiológica y en el último tercio del período de llenado de granos, para tres cultivares de cebada sembrados en cuatro fechas en 2009 y dos fechas en 2011.

\begin{tabular}{|c|c|c|c|c|c|c|c|c|}
\hline Cultivar & Año & $A^{(1)}$ & MF & Siembra & $T_{A-M F}$ & $\mathrm{~T}_{\text {UT }}$ & $P_{\text {A-MF }}$ & $P_{U T}$ \\
\hline & & & & Mes & & & & \\
\hline \multirow[t]{4}{*}{ INIA Ceibo } & 2009 & 25. set. & 4.nov. & May & 15 & 19 & 371 & 108 \\
\hline & 2009 & 8.oct. & 12.nov. & Jun & 17 & 17 & 330 & 20 \\
\hline & 2009 & 23.oct. & 21.nov. & Jul & 18 & 20 & 125 & 0 \\
\hline & 2009 & 30.oct. & 27.nov. & Ago & 19 & 21 & 110 & 25 \\
\hline \multirow[t]{4}{*}{ INIA Guaviyú } & 2009 & 18.set. & 27.oct. & May & 12 & 16 & 229 & 30 \\
\hline & 2009 & 1.oct & 1.nov. & Jun & 16 & 18 & 346 & 105 \\
\hline & 2009 & 14.oct. & 11.nov. & Jul & 17 & 17 & 287 & 20 \\
\hline & 2009 & 23.oct. & 17.nov & Ago & 18 & 20 & 152 & 48 \\
\hline \multirow[t]{4}{*}{ Maltería Pampa } & 2009 & 30.set. & 6.nov. & May & 16 & 20 & 349 & 108 \\
\hline & 2009 & 12.oct. & 14.nov. & Jun & 17 & 18 & 287 & 17 \\
\hline & 2009 & 25.oct. & 23.nov. & Jul & 19 & 20 & 125 & 0 \\
\hline & 2009 & 1.nov. & 29.nov & Ago & 19 & 21 & 48 & 28 \\
\hline \multirow[t]{2}{*}{ INIA Ceibo } & 2011 & 7.oct. & 10.nov. & May & 17 & 20 & 80 & 19 \\
\hline & 2011 & 5.nov & 29.nov. & Ago & 21 & 24 & 66 & 7 \\
\hline \multirow[t]{2}{*}{ INIA Guaviyú } & 2011 & 27.set. & 30.oct. & May & 16 & 16 & 61 & 3 \\
\hline & 2011 & 27.oct. & 20.nov & Ago & 19 & 21 & 60 & 39 \\
\hline \multirow[t]{2}{*}{ Maltería Pampa } & 2011 & 10.oct & 12.nov. & May & 17 & 19 & 41 & 19 \\
\hline & 2011 & 7.nov. & 2. dic & Ago & 21 & 22 & 66 & 7 \\
\hline
\end{tabular}

$\overline{\mathrm{T}}_{\mathrm{A}-\mathrm{MF}\left({ }^{\circ} \mathrm{C}\right):}$ temperatura media desde antesis a madurez fisiológica; $\mathrm{P}_{\mathrm{A}-\mathrm{MF}(\mathrm{mm}):}$ precipitación acumulada desde antesis a madurez fisiológica; $\mathrm{T}_{\mathrm{UT}\left({ }^{\circ} \mathrm{C}\right):}$ temperatura media en el último tercio del llenado de grano); $\mathrm{P}_{\mathrm{UT}}(\mathrm{mm})$ : grano; $A^{(1)}$ : antesis; MF, madurez fisiológica.

2011 las fechas de siembra de mayo registraron en promedio similares $\mathrm{P}_{\mathrm{A}-\mathrm{MF}}(61 \mathrm{~mm})$ que las de agosto $(64 \mathrm{~mm})$. Con relación a las condiciones en el último tercio, en el año 2009 para el cv. IC y el $\mathrm{cv}$. MP las fechas de siembra de mayo registraron en promedio mayores $\mathrm{P}_{\mathrm{UT}}(108 \mathrm{~mm})$ con respecto agosto $(27 \mathrm{~mm})$ mientras que para el cV. IG fueron similares (30-48 mm). En el año 2011 las fechas de siembra de mayo registraron en promedio similares $\mathrm{P}_{\mathrm{UT}}(14 \mathrm{~mm})$ que las de agosto $(18 \mathrm{~mm})$.

El nivel de dormición posterior a madurez fisiológica dependió de la interacción entre fecha de siembra y cultivar $(p<0,0001)$ (Figura 1). En 2009, retrasos en la fecha de siembra incrementaron la germinación para los cvs. IC y MP, mientras que para el cv. IG la germinación se incrementó con siembras de junio respecto a mayo y luego disminuyó, aunque permaneció mayor que los valores de las siembras de mayo.

En 2011 la germinación se incrementó con el atraso en la fecha de siembra para los tres cultivares, debido a que en este año solo se efectuaron dos épocas de siembra, el comportamiento diferente que mostró el cv. IG en el año 2009 no pudo ser evaluado. Estos resultados fueron similares a los reportados por Benitez (1999) y Castro et al. (2010) quienes detectaron que el nivel de dormición adquirido por cada lote de semilla es regulado por el genotipo y las condiciones de ambiente en que se forman las semillas.

Al igual que para el nivel de dormición, la sensibilidad al agua mostró una interacción significativa $(p<0,0001)$ entre el cultivar y las fechas de siembra dentro de cada año. Resultados similares fueron reportados por Castro et al. (2010) quienes mencionaron que al igual que la dormición las condiciones del ambiente durante el llenado de grano y el genotipo condicionan el valor de sensibilidad al agua (Figura 2). Las siembras más tardías en el año 2009 redujeron la sensibilidad al agua para los tres cultivares, aunque en el año 2011 este efecto se registró sólo para el cv. MP.

Las $\mathrm{T}_{\text {A-MF }}$ con las $\mathrm{T}_{\mathrm{UT}} \mathrm{y}$ las $\mathrm{P}_{\mathrm{A}-\mathrm{MF}}$ con las $\mathrm{P}_{\mathrm{UT}}$ presentaron correlaciones positivas y altas $\left(r^{2}=0,84\right.$ y $r^{2}=0,68$, respectivamente). Para ajustar el modelo lineal por cultivar se eligieron las variables ambientales $T_{\text {A-MF y }} P_{\text {A-MF, }}$ que presentaron mejor correlación con la germinación y sensibilidad al agua (Tabla 2). La $T_{U T}$ no fue significativa $(p \leq 0,05)$ sobre la germinación, a diferencia de los trabajos de Castro et al. (2010) que reportaron una estrecha relación entre $T_{U T}$ y el nivel de dormición de los granos. En este sentido, la aplicación de un modelo general para estimar la dormición que incluya el efecto de la $\mathrm{T}_{\mathrm{A}-\mathrm{MF} \text { y }} \mathrm{P}_{\mathrm{A}-\mathrm{MF}}$, la determinación de la antesis y el momento de madurez fisiológica de los genotipos simplifica y facilita su aplicación. 


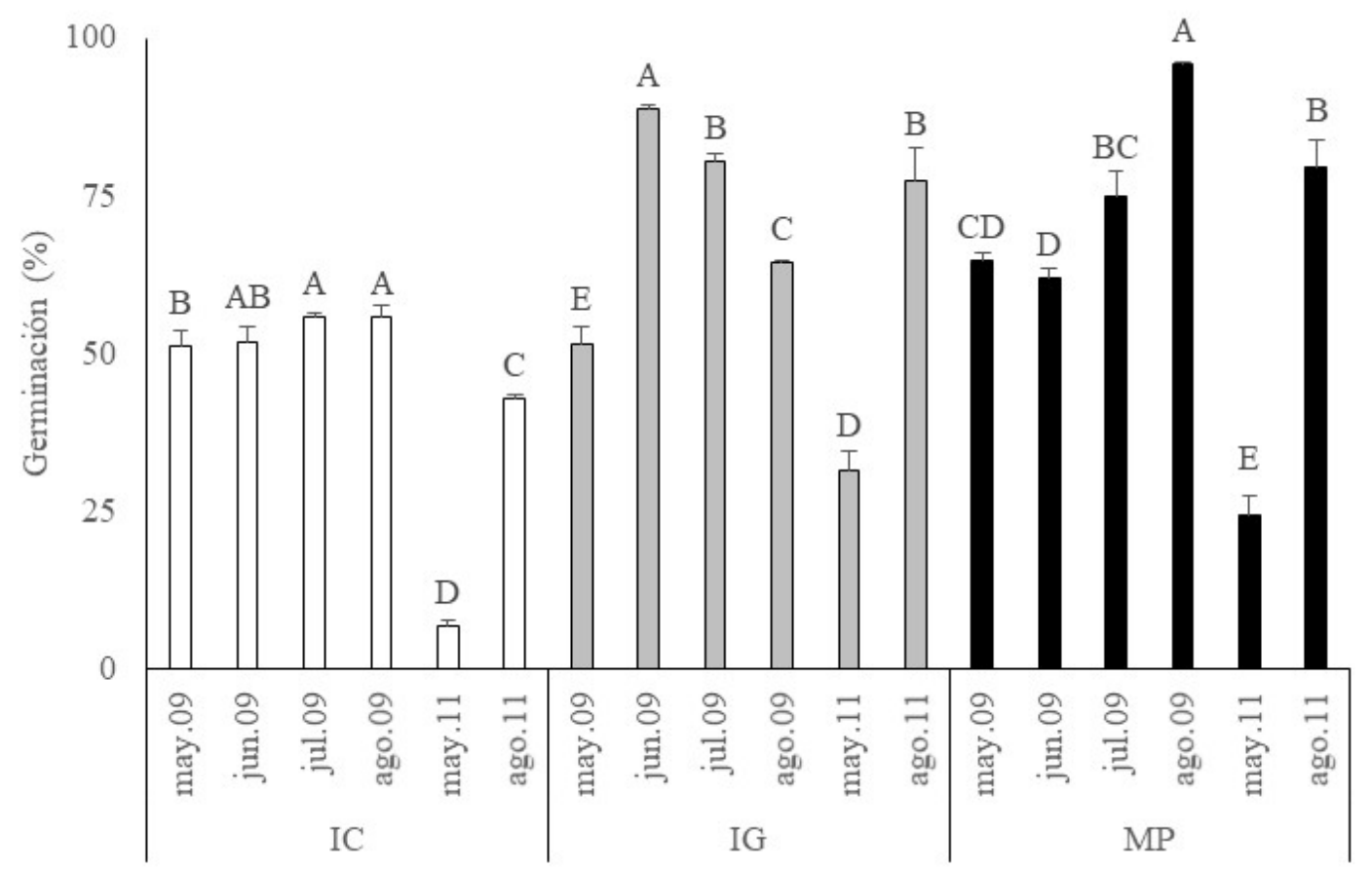

Figura 1. Germinación de los granos de cebada a los 12 días post-madurez fisiológica para los cultivares, INIA Ceibo (IC), INIA Guaviyú (IG) y MP1010 (MP), en cuatro fechas de siembra en 2009 y dos fechas de siembra en 2011. Letras diferentes indican diferencias estadísticas $(p \leq 0,05)$ dentro de cada cultivar. Las barras verticales representan el error estándar.

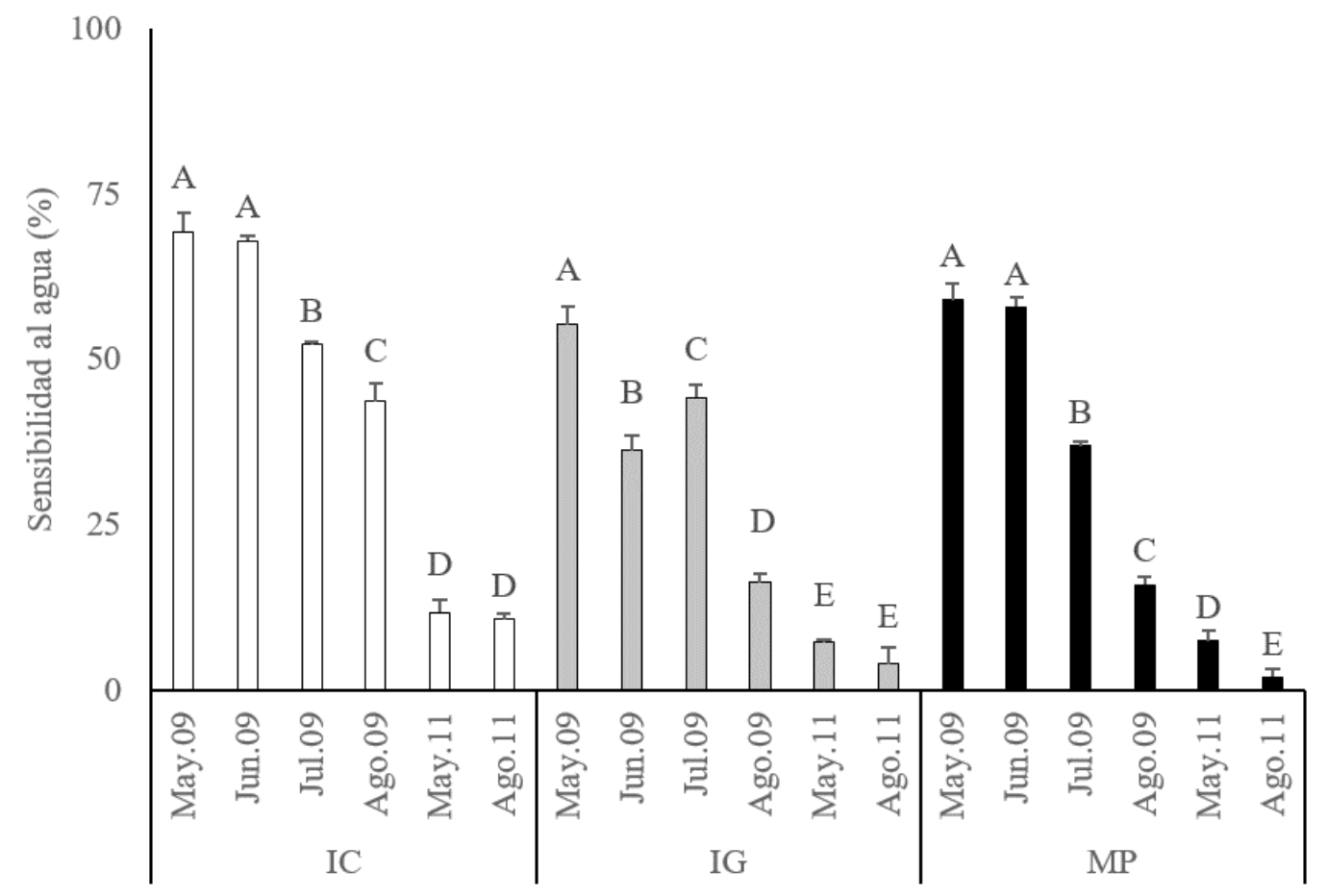

Figura 2. Sensibilidad al agua (\%) de los granos de cebada a los 100 días post-madurez fisiológica para los cultivares INIA Ceibo (IC), INIA Guaviyú (IG) y MP1010 (MP) en cuatro fechas de siembra en 2009 y dos fechas de siembra en 2011. Letras diferentes indican diferencias estadísticas $(p \leq 0.05)$ dentro de cada cultivar. Las barras verticales representan el error estándar 
Tabla 2. Coeficientes de correlación de las variables $T_{A-M F\left({ }^{\circ} C\right)}, P_{A-}$ MF (mm). $T_{U T\left({ }^{\circ}\right)}$ y $P_{U T(m m)}$, para las estimaciones de los valores de germinación y sensibilidad al agua $(n=60)$.

\begin{tabular}{lcccc}
\hline Variable & $\mathrm{T}_{\mathrm{A}-\mathrm{MF}\left({ }^{\circ} \mathrm{C}\right)}{ }^{(1)}$ & $\mathrm{P}_{\mathrm{A}-\mathrm{MF}(\mathrm{mm})}$ & $\mathrm{T}_{\mathrm{UT}(\mathrm{mm})}$ & $\mathrm{P}_{\mathrm{UT}(\mathrm{mm})}$ \\
\hline Germinación & $0,25^{\star *}$ & $0,25^{\star *}$ & $0,12 \mathrm{~ns}$ & $0,23^{\star *}$ \\
$\begin{array}{l}\text { Sensibilidad } \\
\text { al agua }\end{array}$ & $-0,51^{* * *}$ & $0,84^{* * *}$ & $-0,42^{* * *}$ & $0,40^{* * *}$ \\
\hline
\end{tabular}

***significativo al $\mathrm{p} \leq 0,001,{ }^{* *}$ significativo al $\mathrm{p} \leq 0,05$, ns no significativo con $p>0,05$. (1) $T_{A-M F(C):}$ temperatura media desde antesis a madurez fisiológica; $P_{A-M F(m m):}$ precipitación acumulada desde antesis a madurez fisiológica; $T_{\left.U T{ }^{\circ} \mathrm{C}\right)}$ temperatura media en el último tercio de llenado de grano: $P_{U T(\mathrm{~mm})}$ : precipitación acumulada en el último tercio de llenado de grano.

Los modelos lineales para predecir el valor de germinación incluyendo los efectos de $\mathrm{T}_{\mathrm{A}-\mathrm{MF}} \mathrm{y}$ $P_{\text {A-MF }}$ mostraron efectos positivos y significativos de ambas variables sobre el nivel de dormición de las semillas de los tres cultivares evaluados (Tabla 3). Incrementos de la $T_{\text {A-MF }}$ en el rango de 15 a $21^{\circ} \mathrm{C}$ para el cv. IC, 12 a $19^{\circ} \mathrm{C}$ para el cv. IG y 16 a $21^{\circ} \mathrm{C}$ para el $\mathrm{cv}$. MP, se asociaron con una mayor germinación (Tabla 1 y 3) resultado de un menor nivel de dormición, en coincidencia con lo reportado por Benítez (1999).

Por otra parte, incrementos de la $\mathrm{P}_{\mathrm{A}-\mathrm{MF}}$ también aumentaron la germinación en los tres cultivares estudiados (Tabla 3), en coincidencia con Aspinall (1965) y Biddulp et al. (2007), quienes observaron que el déficit hídrico durante el llenado de grano redujo la germinación de las semillas de cebada y trigo, respectivamente. Contrariamente a lo aquí observado, Gualano (2004) detectó que la falta de agua durante el llenado de grano incrementó la germinación si la temperatura durante la ventana de sensibilidad (ubicada en el último tercio del llenado de grano) fue menor a $20^{\circ} \mathrm{C}$, de lo contrario el nivel de dormición dependió principalmente de la temperatura.

Los modelos lineales ajustados para estimar la sensibilidad al agua mostraron que las precipitaciones durante el llenado del grano favorecieron la ocurrencia de valores más altos de sensibilidad al agua en los tres cultivares. Esto podría deberse a una mayor presencia de microrganismos en los granos, según Gabery Roberts, 1969; Briggs y McGuinness, 1993; Noots et al., 1999; Thomas y Usher, 2001; en condiciones de exceso de agua los microorganismos se multiplican rápidamente, limitan la respiración del embrión y en consecuencia inducen sensibilidad al agua.

Sin embargo, la sensibilidad al agua del cv. IG también se incrementó por descensos de la $T_{A-M F}$ (Tabla 3), lo que concuerda con lo observado por Jansson (1962), quien mencionó que la sensibilidad al agua es una forma de dormición que aparece cuando los granos maduran bajo clima húmedo y temperaturas frías. Para los cvs. IC y MP la sensibilidad al agua dependió de las $P_{\text {A-MF }}$ y no varió por efecto de la $T_{A-M F}$ en coincidencia con Woonton et al. (2005) quienes observaron un comportamiento diferente de los genotipos en respuesta a los ambientes durante el crecimiento de los granos.

En el presente trabajo no se encontraron asociaciones entre las variables dormición y sensibilidad al agua; ambas fueron afectadas por el nivel de precipitaciones durante el llenado de grano y por la temperatura de dicho período a excepción del nivel de sensibilidad al agua de los cvs. IC y MP. Estos resultados fueron similares a los reportados por Castro et al. (2010) que además explicaron que si bien ambas variables tienen una base genética común son muy sensibles a las condiciones del ambiente de maduración del grano. En este sentido, Woonton et al. (2005) señalaron que cuando los granos presentan microorganismos, la dormición durante el almacenamiento disminuye; sin embargo, la sensibilidad al agua puede permanecer elevada.

Los resultados obtenidos junto con la expansión de modelos hacia otros cultivares comerciales que incluyan otras variables como calidad sanitaria de los granos, podrán estimar la dormición y

Tabla 3. Valores de $R^{2}$ y parámetros de los modelos lineales; ordenada en el origen (A) y los coeficientes de regresión de las variables $\mathrm{T}_{\mathrm{A}-\mathrm{MF}\left({ }^{\circ} \mathrm{C}\right)}$ y $\mathrm{P}_{\mathrm{A}-\mathrm{MF}(\mathrm{mm}),}$ para las estimaciones de los valores de germinación y sensibilidad al agua para cada cultivar $(\mathrm{n}=20)$.

\begin{tabular}{|c|c|c|c|c|c|c|}
\hline Variable & Cultivar & $\mathrm{R}^{2}$ & A & $T_{A-M E\left({ }^{\circ} \mathrm{C}\right)}{ }^{(1)}$ & $P_{A-M F(m m)}$ & SD \\
\hline \multirow[t]{3}{*}{ Germinación } & $I C^{(2)}$ & 0,55 & $-131,03$ & $8,16^{\star *}$ & $0,16^{\star \star \star}$ & 3,6 \\
\hline & IG & 0,74 & $-72,32$ & $6,78^{\star \star *}$ & $0,15^{\star \star *}$ & 3,3 \\
\hline & MP & 0,56 & $-205,95$ & $13,89^{\star *}$ & $0,13^{\star *}$ & 4,1 \\
\hline \multirow[t]{3}{*}{ Sensibilidad al agua } & IC & 0,74 & $1,90 \mathrm{~ns}$ & $0,39 \mathrm{~ns}$ & $0,18^{\star * *}$ & 3,7 \\
\hline & IG & 0,86 & $7,16^{\star \star \star}$ & $-4,13^{\star \star \star}$ & $0,12^{\star \star \star}$ & 2,8 \\
\hline & MP & 0,88 & $28,81 \mathrm{~ns}$ & $-1,40 \mathrm{~ns}$ & $0,17^{\star \star \star}$ & 2,9 \\
\hline
\end{tabular}

${ }^{* * *}$ significativo al $\mathrm{p} \leq 0,001,{ }^{* *}$ significativo al $\mathrm{p} \leq 0,01$, ns no significativo con $\mathrm{p}>0,05$. (1) $\mathrm{T}_{\left.\mathrm{A}-\mathrm{MF}{ }^{\circ} \mathrm{C}\right)}$. temperatura media desde antesis a madurez fisiológica; $P_{\text {A-MF (mm): }}$ precipitación acumulada desde antesis a madurez fisiológica; SD: desvío estándar. (2) IC INIA Ceibo, IG INIA Guaviyú, MP Maltería Pampa. 
sensibilidad al agua en forma anticipada. En caso de lluvias abundantes durante el llenado de grano permitirá adoptar medidas de manejo como el incremento de la aireación de los granos durante el proceso de malteo y así reducir la sensibilidad al agua.

\section{CONCLUSIONES}

Con temperaturas medias altas durante el llenado de grano es esperable menores valores de dormición; sin embargo, su efecto en la disminución de la sensibilidad al agua dependerá del cultivar. Años con altas precipitaciones durante el llenado de grano provocarían una disminución de la dormición, pero un aumento de la sensibilidad al agua, y consecuentemente, retrasos en el inicio del proceso de malteo. La germinación de las semillas al momento de cosecha no predice la sensibilidad al agua.

\section{AGRADECIMIENTOS}

Al Instituto Nacional de Investigación Agropecuaria (INIA), Uruguay, por financiar esta investigación. El autor quiere agradecer a Ariel Castro por compartir su conocimiento e introducirlo en el tema de estudio.

\section{BIBLIOGRAFÍA}

Arias, G. (1991). Calidad industrial de la Cebada Cervecera. (Serie Técnica 18). Instituto Nacional de Investigación Agropecuaria, Montevideo, Uruguay.

Aspinall, D. (1965). Effects of the soil moisture stress on the growth of barley. Australian Journal of Agricultural Research 16(3) 265 - 275. doi: 10.1071/AR9650265.

Benech-Arnold, R. (2002). Bases of pre-harvest sprouting resistance in barley: Physiology, molecular biology and environmental control of dormancy in the barley grain. En G. Slafer, J. Molina-Cano, R. Savin, J. Araus y I. Romagosa (Eds.), Barley Science Recent advances from molecular biology to agronomy of yield and quality (481-502). New York, United States: The Haworth Press, Inc.

Benech-Arnold, R. (2004). Inception, Maintenance, and Termination of Dormancy in Grain Crops: Physiology, Genetics and Environmental Control. En R. BenechArnold, R. Sánchez (Eds.), Handbook of Seed Physiology: applications to agriculture (169-198). New York, United States, CRC Press.

Benech-Arnold, R., Giallorenzi, C., Frank, J. y Rodriguez, V. (1999). Termination of hull-imposed dormancy in developing barley grains is correlated with changes in embryonic ABA levels and sensitivity. Seed Science Research, 9 (1), 39-47. doi: 10.10171 S09602585990000045.

Benech-Arnold, R.,Gualano, N., Leymarie, J., Côme, D. y Corbineau, F. (2006). Hypoxia Interferes with ABA Metabolism and Increases ABA Sensitivity in Embryos of Dormant Barley Grains. Journalof Experimental Botany, 57(6), 1423-1430. doi: 10.1093/jxb/erj122.

Benítez, A. (1999). Dormancia en semillas de cebada cervecera. Cangüé, 16, 25-31.

Bewley, J. y Black, M. (1994). Dormancy and the Control of Germination. En J. Bewley, M. Black (Eds.), Seeds Physiology of development and germination (199267). New York, United States: Plenum Press.

Biddulph, T., Plummer, J., Setter, T. y Mares, D. (2007). Influence of high temperature and terminal moisture stress on dormancy in wheat (Triticum aestivum L.). Field Crops Research, 103 (2), 139-153. doi: 10.1016/j. fcr.2007.05.005.

Briggs, D. y McGuinness, G. (1993). Microbes and barley grains. Journal of the Institute of Brewing, 99 (3), 249255. doi: 10.1002/j.2050-0416. 1993.tb01168.x.

Castro, A., Benítez, A., Hayes, P.,Viega, L. y Wright, L. (2010). Coincident quantitative trait loci effects for dormancy, water sensitivity and malting quality traits in the BCD47 × Baronesse barley mapping population. Crop and Pasture Science, 61 (9), 691-699. doi: 10.1071/CP10085.

Côme, D. (1998). Germination. En P. Mazliak (Ed.), Croissans et développement. Physiologie Végétale, II, (129-226). París, Francia: Hermann.

Di Rienzo, J., Casanoves, F., Balzarini, M., Gonzalez, L., Tablada, M. y Robledo, C. W. InfoStat versión 2016. Grupo InfoStat. FCA. Universidad Nacional de Córdoba. Argentina. Recuperado de: http://www. infostat.com.ar.

Gaber S. y Roberts, E. (1969). Water-sensitivity in barley seed II. Association with microorganism activity. Journal of the Institute of Brewing, 75 (3), 303-314. doi: 10.1002/j.2050-0416. 1969.tb03216.X.

Gong, X., Li, C., Zhou, M., Bonnardeaux, Y. y Yan, G. (2014). Seed dormancy in barley is dictated by genetics, environments and their interactions, Euphytica, 197(3), 355-368. doi:10.1007/s10681-0141072-X.

Gualano, N. (2004). Brotado pre-cosecha y pregerminado en cebada cervecera: Predicción de la susceptibilidad del cultivo basada en el ambiente y manejo post-cosecha de los granos dañados. Disertación doctoral no publicada, Universidad de Buenos Aires, Buenos Aires, Argentina.

Jansson, C. (1962). Studies on water-sensitivity in barley 
seeds. Svensk Kem. Tidskr, 74, 181-194.

Gong, X., Li, C., Zhou, M., Bonnardeaux, Y. y Yan, G. (2014). $\mathrm{H}_{2} \mathrm{O}_{2}$ mediates the regulation of $\mathrm{ABA}$ catabolism and GA biosynthesis in Arabidopsis seed dormancy and germination. Journal of Experimental Botany, 61 (11), 2979-2990. doi: 10.1093/jxb/erq125.

Millar, A., Jacobsen, J., Ross, J., Helliwell, C., Poole, A., Scofield, G., Reid, J. y Gubler, F. (2006). Seed dormancy and ABA metabolism in Arabidopsis and barley: the role of ABA 8'-hydroxylase. Plant Journal, 45 (6), 942-954. doi: 10.1111/j.1365-313X.2006. 02659.x.

Noots, I., Delcour, J. y Michiels, C. (1999). From field barley to malt: detection and specification of microbial activity for quality aspects. Critical Reviews in Microbiology, 25 (2), 121-153. doi: 10.1080/10408419991299257.

Pararajasingham, S. y Hunt, L. (1991). Wheat spike temperature in relation to base temperature for grain filling duration. Canadian Journal of Plant Science, 71 (1), 63-69. doi: 10.4141/cjps91-007.

Pollock, J. (1962). Barley and malt. En A.H Cook (Ed.), Biology Biochemistry, technology, (pp. 303-399). London, England: Academic Press.

Reuss, R., Cassells, J. y Green, J. (2003). Malting barley: storage, dormancy and processing quality.. En: E.J. Wright, M.C. Webb and E. Highley (Eds.), Australian Postharvest Technical Conference, 1, (4448). Canberra, Australia: Stored Grain Research Laboratory.
Rodriguez, V., Margineda, M., González-Martín, J., Insausti, P. y Benech-Arnold, R. (2001). Predicting pre-harvest sprouting susceptibility in barley: a model based on temperature during grain filling. AgronomyJournal, 93 (5), 1071-1079. doi: 10.2134/ agronj2001.9351071x.

Suburú, G., Gomez, B., Lanaro, V., De Leon, T., Vazquez, D., Castro, M. y Motta, L. (2007). Informe de resultados de la calidad industrial de la evaluación nacional de cebada cervecera. Cosecha 2006. (Informe técnico del Laboratorio Tecnológico del Uruguay). Montevideo, Uruguay: Departamento de Cereales, Oleaginosos y Productos Derivados.

Suburú, G., Gomez, B., Lanaro, V., De Leon, T., Vazquez, D., Castro, M. y Motta, L. (2008). Informe de resultados de la calidad industrial de la evaluación nacional de cebada cervecera. Cosecha 2007. (Informe técnico del Laboratorio Tecnológico del Uruguay). Montevideo, Uruguay: Departamento de Cereales, Oleaginosos y Productos Derivados..

Thomas, K. y Usher, J. (2001). Interactions between lactic acid bacteria and malting barley. Aspects of Applied Biology, 63, 239-250.

Woonton, B., Jacobsen, J., Sherkat, F. y Stuart I. (2005). Changes in Germination and Malting Quality During Storage of Barley. Journal of the Institute of Brewing. 111(1), 33-41. doi: 10.1002/j.2050-0416. 2005. $\underline{\mathrm{tb} 00646 . x}$ 